\title{
Electrocaloric effect in relaxor ferroelectrics
}

\author{
R. Pirc.* Z. Kutnjak, and R. Blinc \\ Jožef Stefan Institute, P.O. Box 3000, 1001 Ljubljana, Slovenia \\ Q. M. Zhang \\ Materials Research Institute and Electrical Engineering Department, \\ The Pennsylvania State University, University Park, PA 16802, USA
}

(Dated: October 25, 2018)

\begin{abstract}
The electrocaloric effect (ECE) in normal and relaxor ferroelectrics is investigated in the framework of a thermodynamic approach based on the Maxwell relation and a Landau-type free energy model. The static dielectric response of relaxors is described by the spherical random bond-random field model, yielding the first Landau coefficient $a=a(T)$, which differs from the usual expression for ferroelectrics. The fourth-order coefficient $b$ is treated as a phenomenological parameter, which is either positive or negative due to the anisotropy of the stress-mediated coupling between the polar nanoregions. When $b<0$, the maximum ECE in a relaxor is predicted near the critical point in the temperature-field phase diagram, whereas in a ferroelectric it occurs at the first order phase transition. The theoretical upper bound on the ECE temperature change is estimated from the values of saturated polarization, effective Curie constant, and specific heat of the material.
\end{abstract}

PACS numbers: 77.70.+a,77.80.Jk,77.84.-s 


\section{INTRODUCTION}

Several articles have recently focused on the electrocaloric effect (ECE) in ferroelectrics and related materials, $\frac{1-\underline{4}}{-}$ which bears analogy with the well known magnetocaloric effect (MCE) $\stackrel{5}{5} \underline{6}$ Here we investigate the mechanisms of ECE in relaxor ferroelectrics, to be referred to as relaxors and normal ferroelectrics (or ferroelectrics), and discuss the specific features of these two groups of materials. In particular, we will discuss the possibility of achieving a giant ECE in bulk inorganic relaxors and ferroelectric materials as well as in organic polymers. These systems offer the prospect of practical applications, such as miniaturized and energy efficient cooling devices, without the need for large electric currents commonly associated with the MCE.

A crucial physical quantity in ECE is the change of entropy of a polar material under the application and/or removal of an external electric field. For example, when the electric field is turned on isothermally, the elementary dipolar entities in the system will become partially ordered and the entropy will be lowered. The entropy lowering of the dipolar subsystem is then compensated by an increase of the temperature of the total system, which characterizes the ECE. The degree of lowering depends on the number of statistically significant configurations in the initial and final states of the system, as well as on the size of the average dipole moment and the volume density of dipolar entities. Other factors may also play a role: If the system undergoes a first order phase transition under the action of external electric field, the entropy will be enhanced on crossing the borderline between the two phases, resulting in a larger ECE. The line of first order transition points terminates at a critical point where the transition becomes continuous, $\underset{7}{ }$ and it will be of special interest to investigate the behavior of ECE in the vicinity of the critical point.

Estimates of the ECE can be made on the basis of thermodynamic Maxwell relations using the measured heat capacity and the field and temperature dependence of the dielectric polarization. From the theoretical point of view, a central problem is how to make predictions about the temperature and field dependence of ECE. As a first step, one needs to develop an appropriate phenomenological and/or mesoscopic model, which incorporates

the specific physical features of the systems. Here we will make use of the standard Landau 
phenomenological model, which can be applied to both relaxors and ferroelectrics with the corresponding choice of Landau coefficients. These in turn can be derived from the mesoscopic model of the material under study. In the case of relaxors, the mesoscopic model of choice is the spherical random bond-random field (SRBRF) model, which is based on the

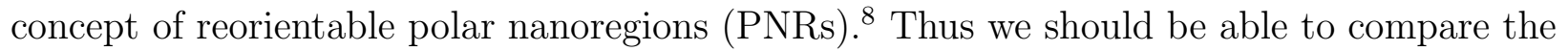
ECE in relaxors and ferroelectrics, and determine the parameters, which control the ECE in these systems. Finally, using general principles of statistical thermodynamics we will discuss the existence of a theoretical upper bound on the ECE and argue that it satisfies a universal relation, which is, in principle, also applicable to MCE.

\section{THERMODYNAMIC APPROACH}

The temperature change of a polar system under adiabatic electric field variation from the initial value $E_{i}=0$ to final value $E_{f}=E$ can be written in the form $\underline{1}$

$$
\Delta T=-\frac{T}{C_{E}} \int_{0}^{E}\left(\frac{\partial P}{\partial T}\right)_{E} d E,
$$

which follows from the thermodynamic Maxwell relation $(\partial S / \partial E)_{T}=(\partial P / \partial T)_{E}$ involving the entropy density $S(E, T)$ and the physical dielectric polarization $P$ (in units of $\mathrm{C} / \mathrm{m}^{2}$ ). The volume specific heat at constant field is given by $C_{E}=\rho c_{E}$.

In deriving Eq. (1), one tacitly assumes that the fluctuations of polarization $P(\vec{r})$ can be ignored and that $P$ represents a thermodynamic variable given by the macroscopic average of $P(\vec{r})$. Furthermore, it is implied that the system is ergodic, i.e., its response time much shorter than the experimental time scale.

If the field and temperature dependence of $P(E, T)$ is known from experiments, the integral in Eq. (1) can be evaluated, yielding an estimate for $\Delta T \stackrel{3 \cdot \underline{4}}{4}$

In model calculations, it seems convenient to change the integration variable in Eq. (1) from $d E$ to $d P(E)$. This is readily done by applying the thermodynamic identity $\underline{9}$

$$
\left(\frac{\partial P}{\partial T}\right)_{E}=-\left(\frac{\partial E}{\partial T}\right)_{P}\left(\frac{\partial P}{\partial E}\right)_{T},
$$

with the result

$$
\Delta T=\frac{T}{C_{E}} \int_{P_{0}}^{P}\left(\frac{\partial E}{\partial T}\right)_{P} d P .
$$


This expression is fully equivalent to Eq. (1), with the new integration limits given by $P_{0}=P(0, T)$ and $P=P(E, T)$.

The partial derivative $(\partial E / \partial T)_{P}$ can be obtained from the free energy density functional $F(P, T)$. Ignoring fluctuations of the order parameter $P$, we write $F$ as a power series

$$
F=F_{0}+\frac{1}{2} a P^{2}+\frac{1}{4} b P^{4}+\frac{1}{6} c P^{6}+\cdots-E P .
$$

This has the standard form of a mean field free energy expansion with temperature dependent coefficients $a, b, c, \ldots$, etc.

Applying the equilibrium condition $(\partial F / \partial P)_{T}=0$, we obtain the equation of state

$$
E=a P+b P^{3}+c P^{5}+\cdots,
$$

and the temperature derivative in Eq. (3) becomes

$$
\left(\frac{\partial E}{\partial T}\right)_{P}=a_{1} P+b_{1} P^{3}+c_{1} P^{5}+\cdots
$$

where $a_{1} \equiv d a / d T, b_{1} \equiv d b / d T$ etc. It should noted be that $P=P(E, T)$ in Eq. (3) is that solution of Eq. (5), which simultaneously minimizes the free energy (44).

The integration in Eq. (3) can now be carried out, yielding

$$
\Delta T=\frac{T}{C_{E}}\left[\frac{1}{2} a_{1}\left(P^{2}-P_{0}^{2}\right)+\frac{1}{4} b_{1}\left(P^{4}-P_{0}^{4}\right)+\frac{1}{6} c_{1}\left(P^{6}-P_{0}^{6}\right)+\cdots\right] .
$$

In passing, we note that $C_{E}$, in general, depends on the temperature; however, in writing down Eqs. (1) and (3) the temperature dependence of the heat capacity had already been ignored.

The expression in brackets is related to the change of the entropy density $S=$ $-(\partial F / \partial T)_{E}$. Using Eq. (4) we can write

$$
S=S_{0}+S_{1}(P)
$$

The first term $S_{0}=-\partial F_{0} / \partial T$ is the entropy at $P=0$. It contains the configuration entropy of dipolar entities, which depends on the number of equilibrium orientations $\Omega$, say, $\Omega=8$ for the $<111>$ equilibrium case $\stackrel{10}{ }^{10}$ Thus we may expect that $S_{0} \sim(N / V) k \ln (\Omega), N$ being the total number of dipolar entities such as PNRs in relaxors. The second term is given by

$$
S_{1}(P)=-\left(\frac{1}{2} a_{1} P^{2}+\frac{1}{4} b_{1} P^{4}+\frac{1}{6} c_{1} P^{6}+\cdots\right) .
$$


Back in Eq. (77), $S_{0}$ cancels out and the ECE temperature change can be rewritten in the familiar form $\underline{\underline{5}}$

$$
\Delta T=-\frac{T}{C_{E}} \Delta S_{P},
$$

with $\Delta S_{P} \equiv S_{1}(P)-S_{1}\left(P_{0}\right)$.

It should be noted that the values of all temperature-dependent quantities $P_{0}, P, a_{1}, b_{1}$, etc., on the r.h.s. of Eq. (7) are taken at the initial temperature $T_{i}=T$, and $C_{E}$ at the final field value $E$.

The coefficients $a, b, c, \ldots$ can be expressed in terms of linear and nonlinear susceptibilities by formally inverting the relation (5) and writing $P(E)$ as a power series in $E \underline{\underline{11}}$ In Landau theory, close to a second order phase transition one sets $a(T) \propto T-T_{0}$, while $b, c, \ldots$ are constants. Thus, $a_{1}=$ const., and $b_{1}=c_{1}=\cdots=0$. This leaves only one nonzero term of the order $\mathcal{O}\left(P^{2}\right)$ in Eq. (7). On the other hand, $\chi_{1} \propto\left|T-T_{0}\right|^{-1}$ and the nonlinear susceptibilities are also found to diverge when $T \rightarrow T_{0}$. Thus a formal inversion $P(E)$ of Eq. (5) in powers of $E$ would lead to a poorly converging series.

In the following we will apply Eq. (7) in order to discuss the predictions of Landau theory in two characteristic cases, namely, normal ferroelectrics and relaxors.

\section{SRBRF MODEL OF ECE IN RELAXORS AND FERROELECTRICS}

As already mentioned, in Landau theory of phase transitions in ferroelectrics, the coefficients $b, c, \ldots$ in Eq. (44) are assumed temperature independent and $a \propto T-T_{0}$, where $T_{0}$ is the Curie-Weiss temperature. When $b>0$ and $E=0$, a second order transition occurs at $T_{c}=T_{0}$. For $b<0$ and $c>0$, a first order transition appears at a temperature $T_{1}$, given by the relation $a\left(T_{1}\right)=3 b^{2} / 16 c$. Writing $a=\left(T-T_{0}\right) /\left(\varepsilon_{0} \Theta\right)$, where $\Theta$ is the Curie constant, we find:

$$
T_{1}=T_{0}+\varepsilon_{0} \Theta \frac{3}{16} \frac{b^{2}}{c} .
$$

For $E \neq 0$, a critical point will be located at $T_{C P}, E_{C P}$, where $\underline{\underline{12}}$

$$
T_{C P}=T_{0}+\varepsilon_{0} \Theta \frac{9}{20} \frac{b^{2}}{c}, \quad E_{C P}=\frac{6 b^{2}}{25 c} \sqrt{\frac{3|b|}{10 c}} .
$$

Turning next to relaxors, we assume that the relevant elementary dipolar entities at temperatures around the dielectric maximum are polar nanoregions or PNRs. According 
to the SRBRF model, $\underline{\underline{8}}$ these PNRs are coupled through Gaussian random interactions $J_{i j}$ ("random bonds") and are subject to Gaussian random fields $h_{i}$. In zero applied field, spontaneous long range order is suppressed $\left(P_{0}=0\right)$. This means that for a relaxor we can still use the free energy (4), however, the coefficient $a(T)$ must remain positive at all temperatures. Thus, for $b>0$ and $E=0$ there can be no second order phase transition. The explicit form of $a(T)$ follows from Eq. (5), namely,

$$
a=\left(\frac{\partial E}{\partial P}\right)_{P=0}=\left(\varepsilon_{0} \chi_{1}\right)^{-1},
$$

where $\chi_{1}=(\partial P / \partial E)_{E=0}$ represents the (quasi)static linear field-cooled dielectric susceptibility. This can be derived from the SRBRF model of relaxors: $\stackrel{*}{*}$

$$
\chi_{1}=\frac{\Theta(1-q)}{T-T_{0}(1-q)},
$$

with

$$
\Theta=\frac{g^{2}}{v k \varepsilon_{0}} .
$$

The effective Curie constant $\Theta$ is given explicitly in terms of the average squared dipole moments $g_{i}$ of PNRs, namely, $g^{2}=\sum_{i=1}^{N} g_{i}^{2} / N$, and the average volume $v=V / N$ associated with a PNR. Later, we will also introduce the saturation polarization $P_{\max }=\bar{g} / v$, where $\bar{g} \equiv \sum_{i=1}^{N} g_{i} / N$. For simplicity, we will henceforth neglect the difference between $\bar{g}$ and $g$, and write $P_{\max } \cong g / v$.

The parameter $\Theta$ can be determined experimentally from the asymptotic high temperature behavior of $\chi_{1} \stackrel{13}{\underline{14}}$ The parameter $T_{0}=J_{0} / k$ is defined via the average over the infinitely ranged random interaction $\left[J_{i j}\right]_{a v}=J_{0} / N$. The spherical glass order parameter, $0<q<1$, is a measure of the degree of disorder. For $P=0$ it is determined by the cubic equation

$$
(k T)^{2} q=\left(J^{2} q+\Delta\right)(q-1)^{2} .
$$

Here, $J^{2}$ is proportional to the variance of the random bond distribution according to $\left[J_{i j}^{2}\right]_{a v}-$ $\left(\left[J_{i j}\right]_{a v}\right)^{2}=J^{2} / N$, while $\Delta$ measures the correlations of quenched random fields, i.e., $\left[h_{i}\right]_{a v}=$ 0 and $\left[h_{i} h_{j}\right]_{a v}=\delta_{i j} \Delta$. If $J_{0}<\sqrt{J^{2}+\Delta}$, long range order will be suppressed $\left(P_{0}=0\right)$. Thus the relaxor state is characterized by three physical parameters: $J_{0}, J$, and $\Delta$. Typically, in relaxors one finds $\Delta \ll J^{2}$, implying that random bonds are effectively much stronger than random fields. This allows PNRs to reorient collectively under the action of external 
fields and relax towards equilibrium. In the opposite case, $\Delta \gg J^{2}$, PNRs are would be trapped in a frozen static configuration of random fields and no characteristic low-frequency relaxor response due to PNR flipping could be observed. Here we will consider only the case $\Delta \ll J^{2}$.

From Eqs. (14) and (13) we derive the coefficient $a(T)$ :

$$
a(T)=\frac{1}{\varepsilon_{0} \Theta}\left(\frac{T}{1-q}-T_{0}\right) .
$$

The linear susceptibility $\chi_{1}$ has been fitted to experimental data for the static field-cooled response in a variety of relaxor systems, from which the parameters of the model have been obtained $\underline{\underline{15}} \underline{\underline{17}}$

Formally, the SRBRF model also yields explicit expressions for nonlinear susceptibilities, from which the coefficients $b$ and $c$ in the free energy (4) can be determined. ${ }^{11}$ However, it has been shown earlier ${ }^{15,16,18}$ that realistic values of these coefficients can only be obtained if the coupling between PNRs and lattice strain fluctuations is included. Several mechanisms for such a coupling have been investigated both at a mesoscopic $\underline{15}, 16,18$ and phenomenological level. ${ }^{19}$ It has also been shown $\frac{18,19}{19}$ that in real three dimensional systems strain coupling gives rise to anisotropy of the anharmonic terms in the free energy $\frac{18}{}$ Specifically, strain coupling may change the sign of the coefficient $b$ and hence of the corresponding nonlinear susceptibility $\chi_{3}$ for a given direction of the applied field. In the following, we will simply consider $b$ and $c$ as free parameters and discuss separately the cases $b>0$ and $b<0$, while keeping $c>0$.

For cubic systems, Eq. (4) can be rewritten in a general form ${ }^{20}$

$$
\begin{aligned}
& F=\alpha_{1} \sum_{i} P_{i}^{2}+\alpha_{11} \sum_{i} P_{i}^{4}+\alpha_{12} \sum_{i \neq j} P_{i}^{2} P_{j}^{2}+\alpha_{111} \sum_{i} P_{i}^{6} \\
& +\alpha_{122} \sum_{i \neq j \neq k} P_{i}^{4}\left(P_{j}^{2}+P_{k}^{2}\right)+\alpha_{123} P_{1}^{2} P_{2}^{2} P_{3}^{2}-\sum_{i} E_{i} P_{i},
\end{aligned}
$$

where $\alpha_{i}, \alpha_{i j}, \alpha_{i j k}$ are so-called dielectric stiffness coefficients with $i, j, k=1,2,3$. For a system with orthorhombic symmetry in a field along [111] we recover Eq. (44), where $P$ is the total polarization, $a=2 \alpha_{1}, b_{[111]}=(4 / 3)\left(\alpha_{11}+\alpha_{12}\right)$, and $c_{[111]}=(2 / 9)\left(3 \alpha_{111}+6 \alpha_{112}+\alpha_{123}\right) . \underline{21}^{2}$ Experiments in $\mathrm{PMN}^{15,22}$ indicate that $\chi_{3}>0$ for $\vec{E} \|[111]$, implying $b_{[111]}<0$. On the other hand, for tetragonal symmetry with $\vec{E} \|[001]$ one has $b_{[001]}=4 \alpha_{11}$ and $c=6 \alpha_{111}$. For example, in $\mathrm{PMN}^{15,22}$ one finds in this case that $\chi_{3}<0$ or $b_{[001]}>0$, indicating that $\alpha_{11}>0$ and $\alpha_{12}<-\alpha_{11}$. 
If $a(T)>b^{2} / c$ at all temperatures, there is no first order phase transition for $E=0$. If $E>0$, however, a first order phase transition with a jump of polarization $\Delta P$ occurs for $E$ exceeding some threshold value $E_{1}$. This can readily be seen by numerical minimization of the free energy (44) for any pair $E, T$. As the temperature increases, $\Delta P(E, T)$ decreases and vanishes at an isolated critical point $T_{C P}, E_{C P}, \underset{7,22}{n}$ where the derivative $(\partial P / \partial T)_{E}$ diverges. 12 The critical temperature is determined from the equation

$$
a_{C P} \equiv a\left(T_{C P}\right)=\frac{9}{20} \frac{b^{2}}{c},
$$

and $E_{C P}$ from the second of Eqs. (12). It should be noted that, generally speaking, $b$ and $c$ can also be functions of $T \underline{7,22}$

For $E>E_{C P}$ the system is in a supercritical regime with continuous temperature and field dependence of $P(E, T)$.

\section{TEMPERATURE AND ELECTRIC FIELD DEPENDENCE OF ECE}

To illustrate the temperature and field dependence of the ECE in relaxors and ferroelectrics, we calculate $\Delta T(E, T)$ from Eq. (7) for a selected set of parameter values. First, we introduce rescaled, dimensionless quantities $F$ and $P$ according to $F \rightarrow F v / J$ and $P \rightarrow P / P_{\max }$, where $P_{\text {max }} \cong g / v$ is the saturation polarization occurring at high field values and/or low temperatures. This requires a rescaling of the remaining parameters according to $a \rightarrow a v /\left(J P_{\max }^{2}\right), b \rightarrow b v /\left(J P_{\max }^{4}\right), c \rightarrow c v /\left(J P_{\max }^{6}\right)$, etc. Also, we redefine $T \rightarrow k T / J$ and $E \rightarrow g E / J$. From Eq. (17) we see that in relaxors the rescaled parameter $a(T)$ behaves as $a(T)=T /(1-q)-T_{0}$, and $a_{1}(T)$ becomes

$$
a_{1}(T)=\frac{\partial}{\partial T}\left(\frac{T}{1-q}\right) .
$$

Here and until the end of this section, the symbols $a, b, c, \ldots$ and $P, T, E$ refer to dimensionless, rescaled parameters, but elsewhere in this paper the same symbols denote the true, physical values of these quantities.

In ferroelectrics, $q=0$ and thus $a(T)=T-T_{0}$, and $a_{1}=1$. In relaxors, in the high temperature limit $T>>1, q$ tends asymptotically to zero, thus $a \sim T-T_{0}$ and $a_{1} \sim 1$, i.e., the same as in ferroelectrics.

In Fig. 1, $a(T)$ and $a_{1}(T)$ are plotted for a relaxor ferroelectric with $J_{0} / J=0.9$ and $\Delta / J^{2}=0.001$. Also shown is the behavior of a normal ferroelectric. It should be noted that 
the essential difference between ferroelectrics and relaxors is the behavior of the corresponding coefficients $a(T)$ and $a_{1}(T)$.

In the following we will choose $b=$ const. $= \pm 1 / 3$ and $c=|b|$. The corresponding ECE temperature change is obtained from Eq. (7). Using the fact that $b_{1}=c_{1}=0$ etc., we find

$$
\Delta T=\frac{k T}{2 v C_{E}} a_{1}(T)\left[P(E, T)^{2}-P_{0}(T)^{2}\right]
$$

The polarization $P(E, T)$ will be calculated numerically by simultaneously solving Eq. (5) and minimizing the free energy (44). We will do that separately for the two cases $b>0$ and $b<0$, assuming the denominator $2 v C_{E}$ to be a constant amplitude factor.

(i) Case $b>0$. As already stated, $P_{0}=P(0, T)=0$ in a relaxor, but in ferroelectrics $P_{0}(T) \neq 0$ for $T<T_{0}$. The spontaneous polarization $P_{0}(T)$ is obtained by minimization of $F(E=0)$. In real systems, $P_{0}$ may not be spatially uniform due to domains. Here we assume that $\left|\vec{P}_{0}\right|$ has the same value in all domains regardless of their orientation, and that the contribution of domain walls to the entropy can be neglected..$^{\underline{5}}$

In Fig. 2(a) we show the calculated values of $\Delta T$ for a relaxor as function of temperature for various values of $E / E_{C P}$, where $E_{C P}$ is formally given by Eq. (12), although the critical point does not exist for $b>0$. Also shown in Fig. 2(b) is $\Delta T$ for a ferroelectric with the same parameters $b, c$, but with different $a$ and $a_{1} . \Delta T$ has a peak at $T_{0}=0.9$ and is in general larger than in the relaxor case. At higher temperatures, however, the difference gradually disappears.

(ii) Case $b<0$. Eqs. (14/16) imply $a(0)=\sqrt{1+\Delta / J^{2}}-J_{0} / J \cong 0.1005$. Thus, $a(0)<3 b^{2} / 16 c$ and the first order phase transition in relaxors at $E=0$ is suppressed. In a ferroelectric, however, a first order transition in zero field occurs at $T_{1}=0.9625$. The critical point is located at $T_{C P}=1.0398$ in relaxors, and at $T_{C P}=1.015$ in ferroelectrics, while $E_{C P}=0.0438$ in both cases. In general, $\Delta T$ is found to increase with increasing field and exhibits a peak as a function of temperature. In relaxors, the peak position moves to higher temperatures with increasing field values, whereas in ferroelectrics the maximum is located at $T=T_{1}$, where a jump of the spontaneous polarization occurs.

In Fig. 3, $\Delta T(T)$ is plotted for four values of the field $\left(E / E_{C P}\right)^{2}$, as indicated. At $E=E_{C P}$ in relaxors, there is a jump of $\Delta T$ due to the field-induced first-order transition. On the other hand, in the case of a ferroelectric, there is a jump of $\Delta T$ at the zero-field first order transition temperature $T_{1}=0.9625$. At the critical point, $\Delta T$ is continuous but with 
an infinite slope in both cases, while at higher temperatures the difference between relaxors and ferroelectrics tends to disappear.

In Fig. 4, the ECE efficiency $\Delta T / E$ is plotted as a function of $E / E_{C P}$ for four values of temperature close to $T_{C P}$. As expected, the maximum efficiency is obtained at the corresponding critical points $T=T_{C P}$ and $E=E_{C P}$.

Larger values of $\Delta T$ in ferroelectrics rather than in relaxors are mainly due to the sharp decrease of $a_{1}(T)$ in relaxors at $T \lesssim 1$ (cf. Fig. 1). However, this does not mean that ferroelectrics are better candidates for achieving giant ECE. Namely, one should bear in mind that there are other parameters, such as $\Theta$ and $C_{E}$, which also have a strong impact on the ECE. Moreover, the above comparison between relaxors and ferroelectrics makes only sense if the coefficients $b, c, \ldots$ are indeed the same in both cases. Therefore, in discussing the ECE in specific systems one should carefully consider the actual physical values of all the relevant model parameters, as discussed in the following section.

\section{APPLICATION TO REAL SYSTEMS}

It has been observed experimentally in a variety of systems that the entropy change $\Delta S_{P}$ in Eq. (10) is proportional to $P^{2} \underline{\underline{23}-25}$ suggesting that the terms of order $\sim P^{4}$ and higher in the expansion (9) make no contribution. Assuming for simplicity that $P_{0}=0$, we recover from Eq. (9) the empirical quadratic relation

$$
\Delta S_{P}=-\frac{1}{2} \beta P^{2}
$$

where the coefficient $\beta$ can be expressed through Eqs. (9) and (17), i.e.,

$$
\beta=a_{1}(T)=\frac{1}{\varepsilon_{0} \Theta} \frac{\partial}{\partial T}\left(\frac{T}{1-q}\right) .
$$

According to the Landau model, in ferroelectrics the partial derivative is equal to 1 or

$\beta=\left(\varepsilon_{0} \Theta\right)^{-1}$ at all temperatures, whereas in relaxors it approaches the value $\sim 1$ at high temperatures, but is in general a function of temperature. Thus, in relaxors, $\beta$ is expected to be a function of temperature with $\beta(T) \leq\left(\varepsilon_{0} \Theta\right)^{-1}$.

Using the relations (10) and (22), the ECE temperature change $\Delta T$ can be written as

$$
\Delta T=\frac{T}{2 C_{E}} \beta P(E, T)^{2} .
$$


A quadratic relation of the same form is predicted by Eq. (21) if the terms $b_{1}, c_{1}, \ldots$ etc. are neglected. It is exactly true in the case $b_{1}=c_{1}=\cdots=0$ discussed in Section III.

Clearly, the above relation is applicable only in the quadratic regime where the empirical Eq. (22) is valid. The parameter $\beta$ can be determined directly from the measured ECE temperature change $\Delta T$, with $P(E, T)$ extracted from dielectric experiments. Alternatively, and especially in ferroelectrics or relaxors at temperatures above the freezing temperature, we can obtain both $P(E, T)$ and the Curie constant $\Theta$ from the dielectric data, and then deduce $\beta$ from Eq. (23). We can then predict $\Delta T$ from Eq. (24). It turns out, however, that the values of $\Delta T$ measured directly may differ from the ones deduced from dielectric data. For example, in the case of relaxor ferroelectric terpolymer P(VDF-TrFE-CFE) at $T \sim 300 \mathrm{~K}$ and $E=70 \mathrm{MV} / \mathrm{M}$, the ECE measured directly is $\Delta T \sim 3.6 \mathrm{~K}$, leading to $\beta \sim 10^{7} \mathrm{~V} \mathrm{~m} \mathrm{C}^{-1} \mathrm{~K}^{-1}, \underline{25}$ whereas the value deduced from dielectric data is $\Delta T \sim 0.87 \mathrm{~K} . \underline{30}$ A tentative explanation of this discrepancy is that even far above the freezing temperature $T_{f} \sim 277 \mathrm{~K}$ the system may still be nonergodic, so that the Maxwell relations, based on equilibrium thermodynamics, are not applicable. ${ }^{30}$ As a consequence, the value of $P(E, T)$ measured on a short time scale is smaller than its thermodynamic long-time limit. Another possibility is that the empirical relation Eq. (22) is an effective quasi-linear relation observed in a broad range of large field values, while its derivation based on the Landau expansion is by assumption restricted to small fields.

We can obtain an estimate for the maximum ECE temperature change $(\Delta T)_{\max }$ by assuming that in a sufficiently strong electric field the polarization reaches its saturation value $P_{\max } \cong g / v$. Relation (22) is not expected to be valid in this saturation regime, and we must return to the general expression (10). Obviously, the expansion (9) cannot be applied due to convergence problems. On the other hand, it is well known $\frac{10}{n}$ that in the saturation regime the excess entropy of the dipolar subsystem tends to zero. Therefore, according to Eq. (8),$S_{1}\left(P_{\max }\right)$ should approach the negative value of the configuration entropy $S_{0}$, i.e., $S_{1}\left(P_{\max }\right) \rightarrow-(k / v) \ln (\Omega)$. Thus, in the saturation regime Eq. (10) leads to

$$
(\Delta T)_{\max }=\frac{k T \ln (\Omega)}{v C_{E}} .
$$

This relation gives the theoretical upper bound on ECE in terms of just three physical quantities, $v, C_{E}$, and the configuration number $\Omega$. Interestingly, this result does not depend explicitly on the dipole moment $g$. Moreover, it does not contain any information about 
possible phase transition occurring in the quadratic regime.

For a given value of electric field $E$, the borderline between the two regimes is expected to occur at some temperature $T^{*}(E)$ where the dipolar energy becomes equal to the thermal fluctuation energy, i.e., $T^{*} \cong g E / k$. For $T \gtrsim T^{*}$ the system is in the quadratic, and for $T \lesssim T^{*}$ in the saturation regime. For the above terpolymer $\mathrm{P}(\mathrm{VDF}-\mathrm{TrFE}-\mathrm{CFE})$, we can estimate the dipole moment $g$ from the relations $P_{\max } \sim 0.1 \mathrm{C} / \mathrm{m}^{2}$ and $g=k /\left(\beta P_{\max }\right)$. Using the value of $\beta$ determined directly from $\Delta T$, we have $g \sim 5.7 \times 10^{-30} \mathrm{C} \mathrm{m}$, and for $E=300 \mathrm{MV} / \mathrm{m}$ we thus find $T^{*} \sim 124 \mathrm{~K}$.

The average volume associated with a PNR in relaxors, $v=V / N$, in Eq. (25) is not a priory known and depends on the total number of PNRs. We can estimate $v$ from the measured values of $\Theta$ and $P_{\max }$ using the relation $\Theta \cong P_{\max }^{2} v / k \varepsilon_{0}$, and rewrite Eq. (25) in the form

$$
(\Delta T)_{\max } \cong \frac{T \ln (\Omega)}{\varepsilon_{0} \Theta C_{E}} P_{\text {max }}^{2} .
$$

The value of $P_{\max }$ can be extracted, for example, from hysteresis loops in the saturation regime, and $\Theta$ from the asymptotic behavior of $\chi_{1}$. As already discussed above, this value of $\Theta$ may differ from the one derived from the experimental value of parameter $\beta \cong\left(\varepsilon_{0} \Theta\right)^{-1}$, observed in ECE experiments in the effective quadratic regime.

In Table I, the predicted values of $(\Delta T)_{\max }$ for a set of selected systems are listed using the values of $\Theta$ deduced from dielectric experiments. It should be noted that Eqs. (25) and (26) provide just a theoretical upper bound for the ECE in the systems listed. In practice, the limit of a fully polarized dipolar subsystem might not be accessible because dielectric breakdown could occur before complete saturation is reached. Nonetheless, the predicted values of $(\Delta T)_{\max }$ permit a comparison between various sytems and might be useful in the search for a giant ECE.

Eq. (25) indicates that a giant ECE is expected to occur in systems with a small value of $v$, or equivalently a large number $N$ of dipolar entities at fixed volume $V$. For illustration, let us consider a specific example, i.e., the ferroelectric copolymer $\mathrm{P}(\mathrm{VDF}-\mathrm{TrFE})$. This system consists of microscopic crystalline layers of polarized material embedded in an amorphous environment $\underline{\underline{31}}$ Electron irradiation breaks up the layered structure into smaller dipolar units, and turns the polymer into a relaxor. Since the number of these new entities is now larger than the number of the original microcrystallites, and assuming the same value of saturation polarization, one expects a stronger ECE to occur in the irradiated relaxor 
copolymer than in the original ferroelectric copolymer. This is corroborated by the experimental values of the coefficient $\beta$ for the irradiated relaxor copolymer $\mathrm{P}(\mathrm{VDF}-\mathrm{TrFE}) \stackrel{30}{\underline{30}}$ $\beta \cong 9.3 \times 10^{7} \mathrm{~V} \mathrm{~m} \mathrm{C} \mathrm{C}^{-1} \mathrm{~K}^{-1}$, and for the original ferroelectric copolymer in the paraelectric phase,$\stackrel{25}{=} \cong 5.8 \times 10^{7} \mathrm{~V} \mathrm{~m} \mathrm{C}^{-1} \mathrm{~K}^{-1}$.

In the case of inorganic relaxor 8/65/35 PLZT thin films, the value of the coefficient $\beta$ is 30 $\beta \sim 1.5 \times 10^{6} \mathrm{~V} \mathrm{~m} \mathrm{C}^{-1} \mathrm{~K}^{-1}$, whereas for the ferroelectric PZT one finds $\frac{1}{\underline{1}} \beta \sim 7.6 \times 10^{5} \mathrm{~V} \mathrm{~m}$ $\mathrm{C}^{-1} \mathrm{~K}^{-1}$. Again, $\beta$ is larger for the relaxor; however, this comparison seems less conclusive since the difference in composition between the two systems is much greater than in the above organic case.

Relation (25) has been derived here in the framework of Landau theory, however, its validity is essentially based on thermodynamic and statistical principles and is hence quite general. In particular, it is independent of any mesoscopic models such as the SRBRF model. Moreover, it can be easily generalized to magnetic systems, where $\Delta T$ represents the MCE temperature change.

The smallest physical limit for $v$ in ferroelectrics is the volume of the unit cell $v_{0}$, yielding the ultimate upper bound on $(\Delta T)_{\max }$ in Eq. (25) for ferroelectric materials. To emphasize this point, let us multiply the numerator and denominator by the Avogadro number $N_{A}$ to obtain

$$
\left(\frac{\Delta T}{T}\right)_{\max }=\frac{R \ln (\Omega)}{C_{m}} .
$$

Here, $R=N_{A} k$ is the gas constant and $C_{m}=N_{A} v_{0} C_{E}$ the molar specific heat. This simple result does not explicitly contain any information on the microscopic nature of the system, i.e., whether it is dielectric, magnetic, etc., since the corresponding electric or magnetic dipole moment does not appear in Eq. (27). Therefore, we can regard the above relation as a universal law for the theoretical upper bound on the ECE and/or MCE in electrically or magnetically polarizable solids. Of course, Eq. (27) implies that the polarization or magnetization must have reached complete saturation. In the dielectric case, this requires large electric fields with the already mentioned possibility of dielectric breakdown. In the magnetic case, however, extremely large fields of the order of $\sim 100 \mathrm{~T}$ may be necessary.

Eq. (27) is valid in the saturation regime $T<T^{*}$, typically at low temperatures, where $C_{m}$ is expected to be temperature dependent. In ferroelectrics, the formation of domains should be taken into account, and the entropy limit $S \rightarrow-S_{0}$ could only be reached in very high fields. In relaxors, there is an additional difficulty that the relaxation of PNRs at 
low temperatures is extremely slow and the above limit can only be reached after very long times.

At high temperatures, $C_{m}$ normally approaches a certain limit. In metals one has $C_{m} \sim$ $3 R$ according to the Dulong-Petit law, which is generally not valid for complex solids. For example, in perovskites, $C_{m} \sim 15 R \cdot \underline{\underline{10}}$ Thus, the maximum ECE in perovskites at high temperatures is of the order $(\Delta T / T)_{\max } \sim 0.0667 \ln (\Omega)$. This limit applies to a large group of perovskites, i.e., paraelectrics, ferroelectrics, relaxors, etc. Specifically, for $\Omega=8$ (see Table I), we find $(\Delta T / T)_{\max } \sim 0.139$. For example, if the system can support large electric fields

such that $T^{*}>300 \mathrm{~K}$, this would lead to a giant ECE temperature change $(\Delta T)_{\max } \sim 40 \mathrm{~K}$ at room temperature. This exceeds roughly by a factor of $\sim 2$ the estimated values for perovskites in Table I, which were derived from the physical values of the parameters $P_{\max }$, $\Theta$, and $C_{E}$.

\section{CONCLUSIONS}

In this paper, we studied the mechanism of ECE in relaxor ferroelectrics (or relaxors) and in normal ferroelectrics (referred to as ferroelectrics). Starting from the widely accepted result for the ECE temperature change $\Delta T(E, T)$, based on the thermodynamic Maxwell relation, we derived an alternative expression which could directly be applied in theoretical model calculations. The results for $\Delta T(E, T)$ have been obtained in two physical regimes of field-temperature variables $E, T$. The first of these is the so-called quadratic regime, where $\Delta T$ is proportional to the square of the dielectric polarization $P(E, T)$, and the second the saturation regime, where $P(E, T)$ is allowed to reach its maximum value $P_{\max }$.

In the quadratic regime, the system can be described by the Landau-type free energy model, in which the harmonic Landau coefficient $a(T)$ depends on the physical nature of the system, i.e., the behavior of $a(T)$ in relaxors differs from that of ferroelectrics. The anharmonic coefficients $b, c, \ldots$, which are common to both cases, then determine the critical behavior of the system in a field $E$. The case $b>0$ does not show any pronounced anomalies. For $b<0$, the polarization $P$ and hence $\Delta T$ rises steeply in a relaxor near the isolated critical point $E_{C P}, T_{C P}$, whereas in a ferroelectric, the largest effect occurs near the first order phase transition in zero field, which is absent in the relaxor case. The ECE efficiency $\Delta T / E$ shows a similar behavior in both cases, and at higher fields the same asymptotic values are found. 
Of course, these conclusions only apply provided that all the remaining physical features, such as the number of equilibrium orientations $\Omega$ of the elementary dipolar entities, etc., are the same in both cases.

Experimentally, the ECE entropy change in the quadratic regime is found to behave as $\Delta S \sim-(1 / 2) \beta P(E, T)^{2}$, which is trivially reproduced by the Landau model. The coefficient $\beta$ tends to have larger values in relaxors, leading to a stronger ECE. In irradiated organic polymer relaxors, this can be explained by the larger number of polar nanoregions (PNRs) and thus a smaller average PNR volume $v$. In the saturation regime, the entropy of the dipolar subsystem generally approaches the negative value of the configuration entropy, $S \sim-(k / v) \ln (\Omega)$, and the maximum ECE value $(\Delta T)_{\max }$ thus crucially depends on the orientational degeneracy $\Omega$.

\section{ACKNOWLEDGMENTS}

This work was supported by the Slovenian Research Agency through Grants P1-0044, P10125, P2-0105, J1-0155, and J1-2015. Research at the Pennsylvania State University was supported by the US DoE, Office of Basic Energy Sciences, Division of Materials Science and Engineering under Award No. DE-FG02-07ER46410. 


\section{References}

* Electronic address: rasa.pirc@ijs.si

1 A. S. Mischenko, Q. Zhang, J. F. Scott, R. W. Whatmore, N. D. Mathur, Science 311, 1270 (2006).

2 A. S. Mischenko, Q. Zhang, R. W. Whatmore, J. F. Scott, N. D. Mathur, Appl. Phys. Lett. 89, $242912(2006)$.

3 T. M. Correia, J. S. Young, R. W. Whatmore, J. F. Scott, N. D. Mathur, Q. Zhang, Appl. Phys. Lett. 95, 182904 (2009).

4 B. Neese, B. Chu, S. G. Lu, Y. Wang, E. Furman, Q. M. Zhang, Science 321, 821 (2008).

5 A. M. Tishin and Y. I. Spichkin, The Magnetocaloric Effect and its Applications (IOP Publishing Ltd., Bristol, UK, 2003).

6 K. A. Gschneidner Jr., V. K. Pecharsky, and A. O. Tsokol, Rep. Prog. Phys. 68, 1479 (2005).

7 Z. Kutnjak, J. Petzelt, R. Blinc, Nature 441, 956 (2006).

8 R. Pirc and R. Blinc, Phys. Rev. B 60, 13470 (1999).

9 H. E. Stanley, Phase Transitions and Critical Phenomena (Oxford University Press, Oxford, UK, 1971), p. 23.

10 Y. Moriya, H. Kawaji, T. Tojo, T. Atake, Phys. Rev. Lett. 90, 205901 (2003).

11 S. Ikeda, H. Kominami, K. Koyama, Y. Wada, J. Appl. Phys. 623342 (1987).

12 M. Iwata, Z. Kutnjak, Y. Ishibashi, R. Blinc, J. Phys. Soc. Japan 77, 034703 (2008).

13 K. Uchino., S. Nomura, L. E. Cross, S. J. Jang, R. E. Newnham, J. Appl. Phys. 51, 1142 (1980).

14 D. Viehland, S. J. Jang, L. E. Cross, M. Wuttig, Phys. Rev. B 46, 8003 (1992).

15 Z. Kutnjak, R. Pirc, and R. Blinc, Appl. Phys. Lett. 80, 3162 (2002).

16 R. Pirc, R. Blinc, Z. Kutnjak, Phys. Rev. B 65, 214101 (2002).

17 V. Bobnar, B. Vodopivec, A. Levstik, Z.-Y. Cheng, Q. M. Zhang, Phys. Rev. B 67, 094205 (2003).

18 R. Pirc, R. Blinc, V. S. Vikhnin, Phys. Rev. B 74, 054108 (2006).

19 R. Pirc, R. Blinc, J. F. Scott, Phys. Rev. B 79, 214114 (2009).

20 A. Amin, R. E. Newnham, L. E. Cross, Phys. Rev. B 34, 1595 (1986).

21 J. H. Qiu, Q. Jiang, Phys. Lett. A 372, 7191 (2008). 
22 Z. Kutnjak, B. Vodopivec, R. Blinc, Phys. Rev. B 77, 054102 (2008).

23 M. Lines, A. Glass, Principles and Applications of Ferroelectrics and Related Materials (Clarendon Press, Oxford, 1977), p. 148.

24 S. G. Lu and Q. M. Zhang, Adv. Mater. 21, 1983 (2009).

25 B. Neese, S. G. Lu, B. Chu, Q. M. Zhang, Appl. Phys. Lett. 94, 042910 (2009).

26 Z. Kutnjak, C. Filipič. A. Levstik, J. Non-Cryst. Solids 305, 398 (2002).

27 S. Sugiyama, A. Takagi, K. Tsuzuki, Jap. J. Appl. Phys. 30, 2170 (1991).

28 S. Kamba, V. Bovtun, J. Petzelt, I. Rychetsky, R. Mizaras, A. Brilingas, J. Banys, J. Grigas, M. Kosec, J. Phys.: Condens. Matter 12, 497 (2000).

29 S. G. Lu, B. Rožič, Q. M. Zhang, Z. Kutnjak, X. Li, E. Furman, L. J. Gorny, M. Lin, B. Malič, M. Kosec, R. Blinc, R. Pirc, J. Appl. Phys. (in the press).

30 S. G. Lu, B. Rožič, Q. M. Zhang, Z. Kutnjak, X. Li, R. Pirc, L. J. Gorny, M. Lin, J. Appl. Phys. (in the press).

31 Q. M. Zhang, Z.-Y. Cheng, V. Bharti, T.-B. Xu, H. Xu, T. X. Mai, S. J. Gross, in Smart Structures and Materials 2000: Electroactive Polymer Actuators and Devices (EAPAD), edited by Y. Bar-Cohen, [Proc. SPIE 3987, 34 (2000)]. 
TABLE I: Predicted limiting values of $(\Delta T)_{\max }$ for various perovskite and polymer relaxor systems according to Eq. (26).

\begin{tabular}{|c|c|c|c|c|c|}
\hline Material & $\Omega$ & $\begin{array}{c}P_{\max } \\
\left(\mathrm{C} / \mathrm{m}^{2}\right)\end{array}$ & $\begin{array}{l}C_{E} \text { at } 300 \mathrm{~K} \\
\left(10^{6} \mathrm{~J} / \mathrm{m}^{3} \mathrm{~K}\right)\end{array}$ & $\begin{array}{c}\beta=\left(\varepsilon_{0} \Theta\right)^{-1} \\
\left(10^{5} \mathrm{Vm} / \mathrm{C} \mathrm{K}\right)\end{array}$ & $\begin{array}{l}\Delta T(\mathrm{~K}) \\
\text { at } 300 \mathrm{~K}\end{array}$ \\
\hline 8/65/35 PLZT & 8 & $0.65^{26}$ & $3.0^{27}$ & $2.43^{28}$ & 21.4 \\
\hline PMN-29.5PT & 8 & $0.55^{7}$ & $2.8^{7}$ & $3.76^{7}$ & 24.8 \\
\hline $\mathrm{PMN}$ & 8 & $0.55^{22}$ & $2.6^{10}$ & $3.82^{14}$ & 20 \\
\hline $\begin{array}{c}\mathrm{P}(\mathrm{VDF}-\mathrm{TrFE}) 68 / 32 \\
\quad \text { (irradiated film) }\end{array}$ & 8 & $0.06^{29}$ & $2.34^{29}$ & $4.67^{29}$ & 39 \\
\hline
\end{tabular}




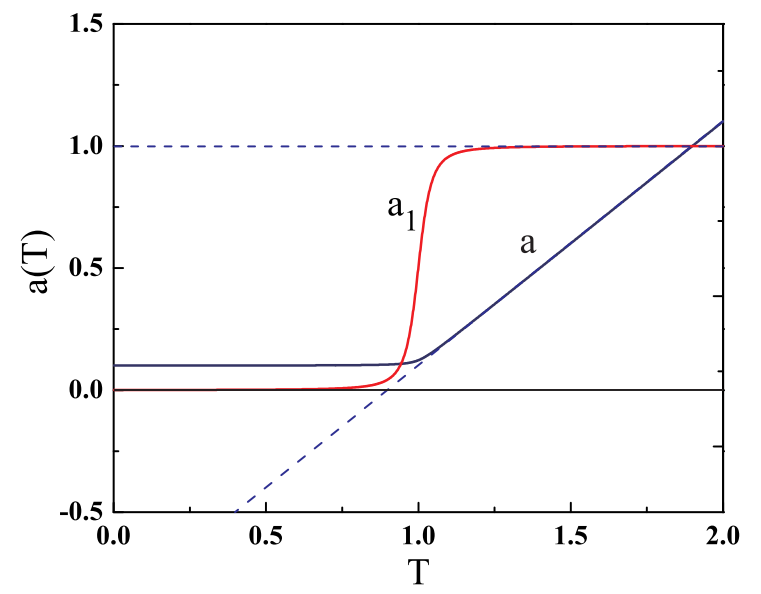

FIG. 1: Temperature dependence of the coefficient $a(T)$ and its temperature derivative $a_{1}(T)$ for a relaxor (solid lines) and a ferroelectric (dashed).

(a)

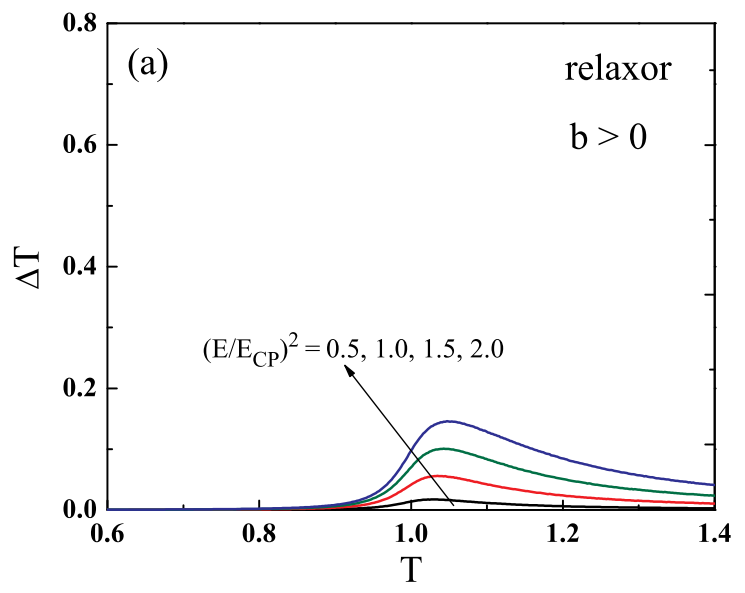

(b)

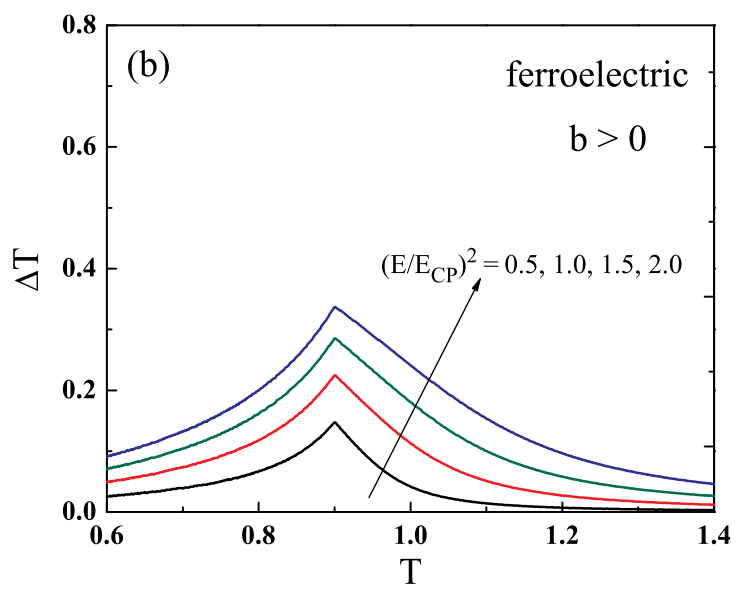

FIG. 2: (a) Calculated temperature dependence of $\Delta T$ in a relaxor with $b>0$ and four values of electric field $E$, as indicated. (b) Same, but for a ferroelectric. 
(a)

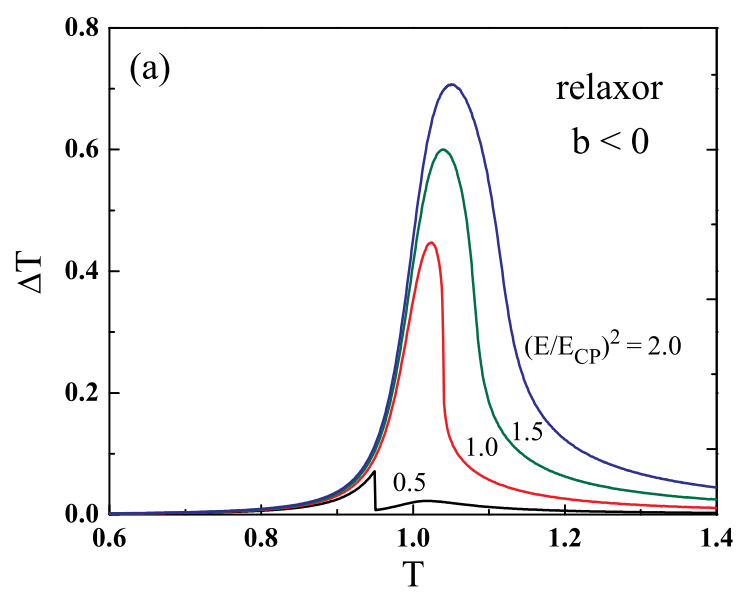

(b)

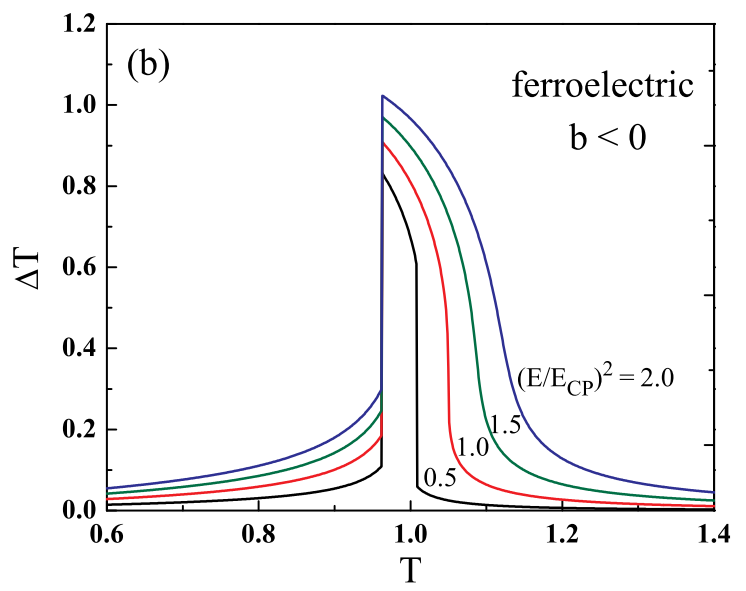

FIG. 3: (a) Calculated temperature dependence of $\Delta T$ in a relaxor with $b<0$ and four values of electric field $E$, plotted on the same vertical scale as in Fig. 1. The critical point lies at $T_{C P}=1.0398$ and $E_{C P}=0.0438$. (b) Same, but for a ferroelectric with $T_{C P}=1.05$ and $E_{C P}=0.0438$, on different vertical scale. Note the first order phase transition at $T_{1}=0.965$.

(a)

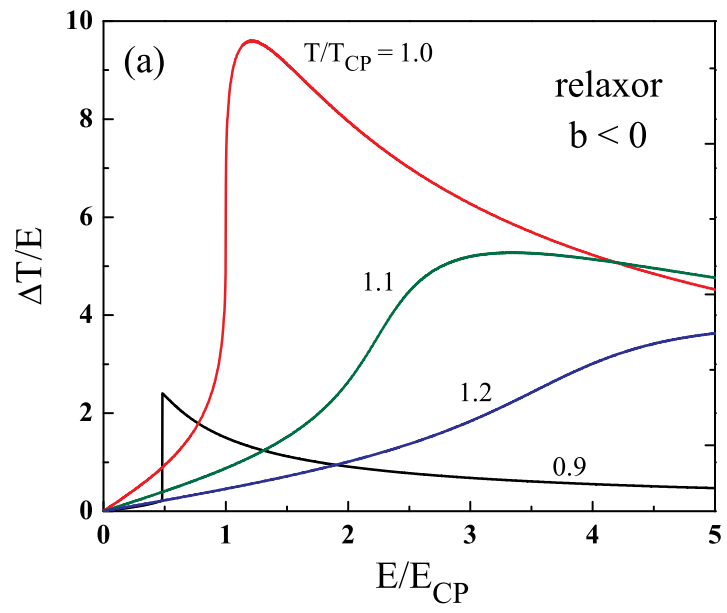

(b)

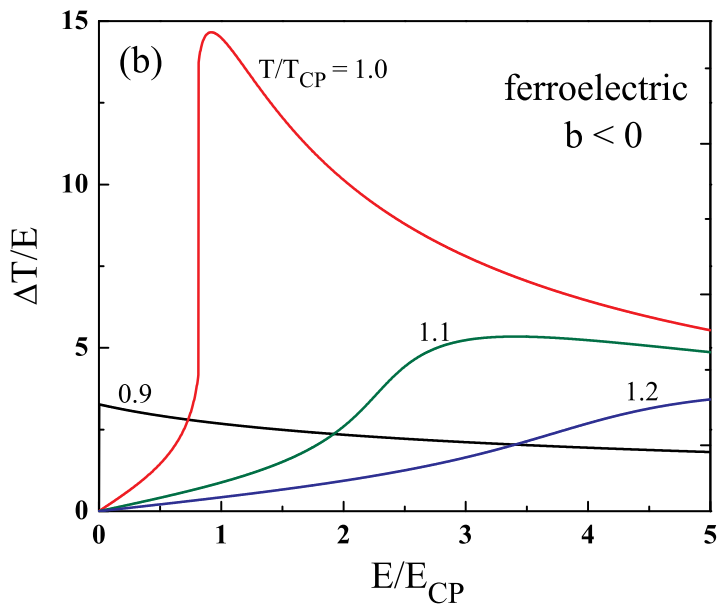

FIG. 4: (a) Calculated field dependence of ECE efficiency $\Delta T / E$ in a relaxor with $b<0$, at four temperatures close to critical temperature $T_{C P}$. (b) Same, but for a ferroelectric, with different vertical scale. 